\title{
Visual cliff preferences following lesions of the visual neocortex in cats and rats'
}

\author{
PATRICIA M. MEYER, ROY A. ANDERSON AND MARY GOOLSBY BRAUN
}

THE OHIO STATE UNIVERSITY

Normal cats, cats with lesions of the visual neocortex, normal rats, enucleated rats, and rats with lesions of the visual neocortex were tested on Gibson's visual cliff for visual depth perception. All animals preferred the shallow side except rats that were enucleated and rats that sustained visual neocortic al ablations.

Much research in the area of visual depth perception has been stimulated by the classic experiment of Walk, Gibson, \& Tighe (1957). By means of the visual cliff technique, these authors concluded that rats could discriminate distance and that this ability to perceive visual depth was probably innate. Since Smith (1937) reported that optic nystagmus, presumably another innate visual response, was present in cats after removal of the visual cortex, Meyer (1963) assessed the cortical dependency of depth perception in cats with extensive neocortical ablations. As measured by the visual cliff, the neodecorticated animals avoided the deep side as often as normal cats. The present experiment replicated the Meyer study (1963) and extended it to determine if the result could be obtained in another species.

Method

Cat. The Ss were eight adult cats. Four served as normal controls and four were subjected to lesions of the visual neocortex.

The visual cliff apparatus was constructed within a $24 \times 48 \times 30$ in. plywood box topped with a sheet of glass. The interior walls and floors contained $3 \times 3$ in. black and white checks, and a trapezoidal starting platform, $6 \mathrm{in}$. high, was centered on the glass (deep side). The floors were transilluminated from below with controlled light intensities to match luminous flux. A trial was initiated by centering $S$ on the platform, and a response was recorded when both front paws contacted the glass surface. $S$ received one trial each day for eight days. The right-left position of the deep side was alternated according to a random series, and data were analyzed by a Mann-Whitney U test.

Rat. Eighty adult rats, 90-120 days old, were divided randomly into three groups. Twenty Ss were normal controls; 20 were enucleated bilaterally; 40 received bilateral lesions of the visual neocortex.

The rat cliff measured $18 \times 38 \times 18$ in. It also was covered with glass, which was divided by a 3 in. wide and 3 in. high starting platform. The walls and floor were covered with $1 / 2$ in. sq. black and white checked wallpaper, and illumination was from below through the cliff floor. The initiation of each trial and the response criterion were the same as for the cat Ss. If the rat Ss did not respond within $5 \mathrm{~min}$., a "no response" trial was recorded, but only five such responses occurred.

The Ss were run one trial per day for three days, and were exposed to the following three lighting conditions: (1) shallow-side-brighter $(0.9 \mathrm{ft} .-\mathrm{lamb}$. vs. $0.5 \mathrm{ft} .-$ lamb.); (2) deep-side-brighter $(0.9 \mathrm{ft} .-1 \mathrm{amb}$. vs. $0.5 \mathrm{ft} .-\mathrm{lamb}$ ); (3) both-sides-equal (0.9 ft.-lamb. vs. $0.9 \mathrm{ft} .-\mathrm{lamb}$ ). The presentation of the lighting conditions was distributed such that each of the three conditions occurred $1 / 3$ of the time on each test day, and following completion of the experiment, all conditions had appeared equally often. The brightness measures were approximated at the rat's eye level near the starting platform by means of a Weston light meter. The apparatus was rotated to eliminate leftright position responding, and the data were analyzed by chi square tests.

Surgery and Histology. Cat surgery was performed aseptically by subpial aspiration of Visual areas I and II, while Ss were under sodium pentobarbital anesthesia. The visual neocortex of rat Ss also was removed by suction, but no sterile precautions were taken.

Following behavior tests, all Ss with cortical lesions were perfused with $0.9 \%$ saline followed by $10 \%$ formalin, and the brains were embedded in celloidin. They were sectioned at $30 \mu$, stained with cresyl violet and analyzed for retrograde degeneration in the dorsal lateral geniculate nuclei (LGN).

Sixteen rats sustained lesions that resulted in complete degeneration of the LGN. Since no behavioral differences were noted between Ss with either complete or incomplete lesions, all were included in the data analysis.

Histological analysis of cat $\mathrm{V}-22$ revealed complete retrograde degeneration in the LGNs, extensive degeneration in the pulvinar nuclei, and unusually enlarged lateral ventricles. Cats $\mathrm{V}-51$ and $\mathrm{V}-27$ showed some sparing that was restricted primarily to the posterior, dorso- and ventro-lateral LGN. The amount of sparing in the left and right LGN was approximately 20 and $21 \%$, respectively, for $\mathrm{V}-51$, and 65 and $25 \%$, respective$1 y$, for $\mathrm{V}-27$. Unfortunately, cat $\mathrm{V}-46$ died prior to perfusion.

\section{Results}

The cat data are summarized in Table 1. Note that all operated and normal Ss responded to the shallow side on Day 1 . Three normal Ss continued to respond to the shallow side, but $\mathrm{N}-60$ developed a position 
Table 1. Visual Cliff Responses

\begin{tabular}{lccccccccc} 
& \multicolumn{4}{c}{ Normal rats } & \multicolumn{4}{c}{ Visual Cats } \\
& N-3 & N-26 & N-39 & N-60 & V.22 & V-27 & V.46 & V-51 \\
\hline Initial Choice & S & S & S & S & S & S & S & S \\
Shallow Side $(\%)$ & 100 & 100 & 100 & 75 & 87.5 & 62.5 & 100 & 100 \\
\hline
\end{tabular}

tendency after Day 3. Although one operated cat responded to both sides equally often after Day 1 , no differences were observed between the two groups.

The rat data are summarized in Table 2. On Day 1 , the Normal Group chose the shallow side more frequently than the deep $(p=.025)$, but on subsequent days, they responded to both sides equally often. The Enucleate Group performed at chance levels throughout the experiment. None of the lighting conditions biased the choices of either group. For the first two test days, the Visual Group could not be distinguished from the Enucleate Group. Curiously, on Day 3, rats with visual lesions selected the deep side more frequently than the shallow $(p<.01)$, but the result could not be accounted for either in terms of position responding or brightness preferences on that test day.

\section{Discussion}

Both normal cats and those with lesions of the visual neocortex avoided the deep side of the visual cliff a finding similar to that obtained by Meyer (1963) in cats with more extensive neocortical ablations.

Table 2. Percent Responses to Shallow Side

\begin{tabular}{lccc} 
& Normal Rats & Enucleated Rots & Visual Rats \\
\hline Day 1 & 75 & 55 & 47.5 \\
Days 2 \& 3 & 55 & 45 & 36.3 \\
\hline
\end{tabular}

However, rats with visual lesions behaved like control animals that had been surgically blinded; they showed no preference for either side during the first two test days.

The fact that both species were not maintained under equivalent living conditions, might possibly account for the rat-cat discrepancy. Prior to surgery, rats were housed in group cages, but following surgery for a three week recovery interval, they were placed in more restricted, $7 \times 9-1 / 2 \times 7$ in. individual cages. In contrast, the cats pre- and postoperatively, lived in large outdoor $4 \times 8 \times 7 \mathrm{ft}$. group wire mesh cages, that contained boxes, which provided an opportunity for jumping and climbing. Since it has been reported (McGuigan, Loizos, \& Carr, 1963) that cliff preferences can be modified by previous proprioceptive experience, it is conceivable that environmental experience following surgery could have influenced cliff choices. On the other hand, it is entirely possible that the difference in cliff preference between rats and cats with visual lesions is one of species.

\section{References}

McGuigan, D. I., Loizos, C., \& Carr, W. J. The effects of previous visual and proprioceptive experience in rat upon performance on the visual cliff. Paper read at Annual Meeting, Eastern Psychological Association, New York, April, 1963.

Meyer, P. M. Analysis of visual behavior in cats with extensive neocortical ablations. J. comp. physiol. Psychol., 1963, 56 397-401.

Smith, K. U. The postoperative effects of removal of the striate cortex upon certain unlearned visually controlled reactions in the cat. J. genet. Psychol., 1937, 50, 137-156.

Walk, R. D., Gibson, E, J., \& Tighe, T. J. Behavior of light- and dark-reared rats on a visual cliff. Science, $126,80-81$.

\section{Note}

1. Supported by NIH Grant MH 06211. 